\title{
Effects of Anti-inflammatory Biflavonoid, Ginkgetin, on Chronic Skin Inflammation
}

\author{
Hyun Lim, ${ }^{a}$ Kun Ho Son, ${ }^{b}$ Hyeun Wook CHang,${ }^{c}$ Sam Sik Kang, ${ }^{d}$ and Hyun Pyo Kim ${ }^{*}, a$ \\ ${ }^{a}$ College of Pharmacy, Kangwon National University; Chunchon 200-701, Korea: ${ }^{b}$ Department of Food and Nutrition, \\ Andong National University; Andong 760-749, Korea: ${ }^{c}$ College of Pharmacy, Yeungnam University; Gyongsan 712-749, \\ Korea: and ${ }^{d}$ Natural Products Research Institute, Seoul National University; Seoul 110-460, Korea. \\ Received January 27, 2006; accepted February 22, 2006
}

Ginkgetin, a biflavonoid from Ginkgo biloba leaves (Ginkgoaceae), was previously demonstrated to inhibit phospholipase $A_{2}$ and to suppress proinflammatory gene expression such as cyclooxygenase-2 (COX-2) and inducible nitric oxide synthase. In this study, the effects of ginkgetin were examined on an animal model of chronic skin inflammation and proinflammatory gene expression. When topically applied to ICR mouse ear, ginkgetin $\left(20-80 \mu \mathrm{g} /\right.$ ear/treatment) inhibited ear edema $(22.8-30.5 \%)$ and prostaglandin $\mathbf{E}_{2}$ production $(30.2-31.1 \%)$ induced by multiple treatment of 12-O-tetradecanoylphorbol-13-acetate (TPA) for 7 consecutive days. By histological comparison, ginkgetin was also found to reduce epidermal hyperplasia. The expression of proinflammatory gene, interleukin-1 $\beta$, was suppressed by ginkgetin. From the results, it is suggested that ginkgetin may be beneficial against chronic skin inflammatory disorders like atopic dermatitis.

Key words ginkgetin; biflavonoid; skin inflammation; gene expression

Although the etiology of chronic skin inflammatory disorders including psoriasis and atopic dermatitis (AD) is not clearly understood, many studies have shown that various proinflammatory enzymes/cytokines play an important role in these inflammatory diseases. For example, phospholipase $\mathrm{A}_{2}\left(\mathrm{PLA}_{2}\right)$ was highly expressed in some psoriatic tissues. ${ }^{1)}$ In addition to lipoxygenase products, ${ }^{2)}$ cyclooxygenase $(\mathrm{COX})$ and prostaglandins (PG) were involved in dermal inflammation and skin wound healing. ${ }^{3,4)}$ Moreover, it was found that high concentrations of cytokines, such as tumor necrosis factor (TNF)- $\alpha$, and adhesion molecules, including intercellular adhesion molecule (ICAM)-1, were deeply involved in the regional site of chronic skin inflammation. ${ }^{5,6}$ Recent studies have also indicated that inducible nitric oxide synthase (iNOS) may be associated with psoriasis, and its reaction product, $\mathrm{NO}$, is involved in various skin disorders. $\left.{ }^{7,8}\right)$ Therefore, it is reasonably thought that an interference of activity and/or expression of these proinflammatory molecules may result in attenuation of chronic skin inflammatory syndromes, and the agents acting on these points may give beneficial effects.

Biflavonoids are flavonoid-flavonoid dimers and some of them possess anti-inflammatory activity. For instance, several biflavonoids including amentoflavone, ginkgetin and isoginkgetin inhibited histamine release. ${ }^{9)}$ Amentoflavone and tetrahydroamentoflavone were found to inhibit COX-1 and/or COX-2. ${ }^{10,11)}$ Some biflavonoids were revealed to suppress the expression of proinflammatory molecules such as COX-2 and iNOS. ${ }^{12,13)}$ These previous investigations have shown the potential of certain biflavonoids for anti-inflammatory agents. In particular, ginkgetin (Fig. 1) mainly found in Ginkgo biloba leaves (Ginkgoaceae) was previously demonstrated as an inhibitor of group IIA sPLA $_{2}$ inhibitor $^{14)}$ and $\mathrm{cPLA}_{2}{ }^{15)}$ In addition, ginkgetin was reported to suppress COX-2 expression from lipopolysaccharide (LPS)-treated macrophages without affecting COX-2 activity, and in vivo study has also revealed that ginkgetin inhibited COX-2 expression and $\mathrm{PGE}_{2}$ production from mouse skin induced by 3 -d treatment of 12-O-tetradecanoylphorbol-13-acetate
(TPA). ${ }^{16)}$ All these findings strongly suggest that ginkgetin may show an inhibition against inflammatory disorders, especially skin inflammation by topical application. Thus, for further characterization of the pharmacological property, the effects of ginkgetin were examined on an animal model of chronic skin inflammation and proinflammatory gene expression in the present investigation.

\section{MATERIALS AND METHODS}

Chemicals TPA and prednisolone were obtained from Sigma-Aldrich Co. (St. Louis, MO, U.S.A.). Ginkgetin was isolated from the methanol extract of Ginkgo biloba leaves according to the previously described procedure. ${ }^{17)}$ The purity was $>95 \%$ based on HPLC analysis.

Animals Male ICR mice (4 weeks, specific pathogenfree) were obtained from Orient Co. (Korea). Animals were fed with laboratory chow (Purina Korea) and water ad libitum. They were acclimatized in an animal facility (KNU) under the conditions of $20-22{ }^{\circ} \mathrm{C}, 40-60 \%$ relative humidity and $12 \mathrm{~h} / 12 \mathrm{~h}$ (light/dark) cycle at least $7 \mathrm{~d}$ prior to experiment.

Chronic Skin Inflammation On day of the experiment (day 1), TPA ( $1 \mu \mathrm{g} / 20 \mu \mathrm{l}$ acetone) was applied to the inner and outer surfaces of mouse ear for inducing a chronic type of skin inflammation according to the original procedure ${ }^{18)}$ with some modification. Test compounds dissolved in oilbased vehicle were topically applied to the same site $(20 \mu \mathrm{l})$ at 1 and $12 \mathrm{~h}$ after TPA treatment. TPA-treated control group only received TPA and vehicle. On day $2-6$, the same treat-

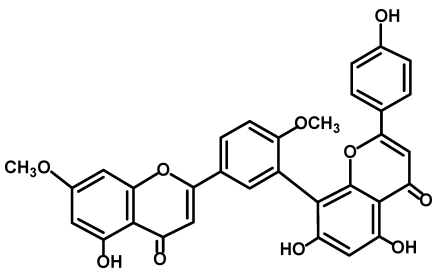

Fig. 1. Chemical Structure of Ginkgetin 
ment regimen was carried out with TPA and test compounds. On day 7, TPA was applied, and one hour later, test compounds were treated. Three randomly selected mice/group were sacrificed by cervical dislocation $3 \mathrm{~h}$ after final treatment of test compounds. Ears were removed and stored in RNA stabilization reagent (Qiagen, Germany) at $-20^{\circ} \mathrm{C}$ for reverse transcriptase-polymerase chain reaction (RT-PCR) analysis. For determination of anti-inflammatory activity, ear thickness of the remaining five mice/group was measured using an engineering gauge (Mitutoyo, Japan) $5 \mathrm{~h}$ after final treatment of test compounds. Immediately after, in order to determine $\mathrm{PGE}_{2}$ concentration and to prepare histological samples, ears were removed. For evaluation of the effects on normal skin, test compounds in the vehicle were treated topically to ears of mice using the same treatment schedule as above without TPA treatment. In this case, the control group received acetone and vehicle only. After $7 \mathrm{~d}$, ear thickness of the test compound-treated groups was measured, but no noticeable difference was observed between the treated and the control groups.

RT-PCR Analysis After cutting into small pieces, ear samples were homogenized in RLT buffer containing 1\% $\beta$-mercaptoethanol for $30 \mathrm{~s}$ using Polytron homogenizer. Total RNA was extracted using RNeasy mini kit (Qiagen) according to the supplier's protocol. The concentration of RNA content was determined by measuring the absorbance at 260 and $280 \mathrm{~nm}$. cDNAs were synthesized using RT reaction at $42^{\circ} \mathrm{C}, 50 \mathrm{~min}$ and $99^{\circ} \mathrm{C}, 5 \mathrm{~min}$ in Gene Cycler thermal cycler (Bio-Rad). Primers were synthesized on the basis of the repeated mouse cDNA sequence for $\mathrm{COX}-1, \mathrm{COX}-2$, interleukin-1 $\beta$ (IL- $1 \beta$ ), TNF- $\alpha$, iNOS, ICAM-1, fibronectin and G3PDH. The primer sequences used for PCR were as follows: COX-1 sense, 5'-TGC ATG TGG CTG TGG ATG TCA TCA A-3', antisense, 5'-CAC TAA GAC AGA CCC GTC ATC TCC A-3', 450 bp; COX-2 sense, 5'-ACT CAC TCA GTT TGT TGA GTC ATT C-3', antisense, 5'-TTT GAT TAG TAC TGT AGG GTT AAT G-3', 583 bp; IL- $1 \beta$ sense, 5'-TGC AGA GTT CCC CAA CTG GTA CAT C-3', antisense, 5'-GTG CTG CCT AAT GTC CCC TTG AAT C3', 387 bp; TNF- $\alpha$ sense, 5'-ACA AGC CTG TAG CCC ACG-3', antisense, 5'-TCC AAA GTA GAC CTG CCC-3', $428 \mathrm{bp}$; iNOS sense, 5'-CCC TTC CGA AGT TTC TGG CAG CAG C-3', antisense, 5'-GGC TGT CAG AGC CTC GTG GCT TTG G-3', 469 bp; ICAM-1 sense, 5'-TCG GAG GAT CAC AAA CGA AGC-3', antisense, 5'-AAC ATA AGA GGC TGC CAT CAC G-3', 471 bp; fibronectin sense, 5'-GCA ACG TGT TAT GAC GAT GG-3', antisense, 5' CTA ACG GCA TGA AGC ACT CA-3', 253 bp; G3PDH sense, 5'-TGA AGG TCG GTG TGA ACG GAT TTG GC3', antisense, 5'-CAT GTA GGC CAT GAG GTC CAC CAC-3', 983 bp. PCR was carried out for 25-30 cycles under saturation, in $25 \mu \mathrm{l}$ reaction mixture. After amplification, $5 \mu \mathrm{l}$ of reaction mixture was analyzed on $1.5 \%$ agarose gel electrophoresis. The bands were visualized by ethidium bromide staining for $10 \mathrm{~min}$. The band density was quantified by densitometric scanning using SigmaGel (Version 1.0, Jandel Sci.). The signal intensities were normalized by comparison with that of G3PDH and represented as relative ratios.

Measurement of $\mathbf{P G E}_{2}$ Concentration As an index of skin $\mathrm{PLA}_{2}$ and/or COX activity, $\mathrm{PGE}_{2}$ concentration was measured essentially following the previously described pro- cedure. ${ }^{16)}$ In brief, the biopsies were obtained by $4 \mathrm{~mm}$ punch from four randomly selected ear samples/group. Then, they were homogenized in $100 \mathrm{~mm}$ phosphate buffer ( $\mathrm{pH}$ 7.4) containing $1 \mathrm{~mm}$ EDTA and $10 \mu \mathrm{M}$ indomethacin. After centrifugation at $1500 \boldsymbol{g}$ for $10 \mathrm{~min}, 50 \mathrm{~mm}$ of citrate buffer $(\mathrm{pH} 3.5)$ was added to the supernatant. The mixture was centrifuged again at $2500 \boldsymbol{g}$ for $10 \mathrm{~min}$. The resulting supernatant was applied to a $6 \mathrm{ml}$ Sep-Pak $\mathrm{C}_{18}$ cartridge (Waters Associate, U.S.A.) and eluted with $5 \mathrm{ml}$ ethyl acetate containing $1 \%$ methanol. The eluent was dried under $\mathrm{N}_{2}$ stream and $\mathrm{PGE}_{2}$ concentration was measured with an ELISA kit (Cayman Chem.) according to the manufacturer's instruction.

Histology Ear samples were fixed in 10\% neutral buffered formalin, embedded in paraffin, sectioned and stained with haematoxylin and eosin (H\&E) based on the standard procedures.

Statistical Analysis All results were represented as arithmetic mean \pm S.D. One-way ANOVA and Student's $t$-tests were used for evaluation of a statistical significance.

\section{RESULTS}

Seven day multiple treatment of TPA on ICR mouse ear induced a chronic type of skin inflammation, characterized by edema, epidermal hyperplasia and infiltration of inflammatory cells (Figs. 2a,b). By histological comparison, ginkgetin was found to considerably reduce these responses (Fig. 2c). Prednisolone used as a reference drug reduced these responses more profoundly (Fig. 2d). When the ear thickness was measured, more than twice increase was observed compared with those of non-treated mice $(0.475 \pm$ $0.031 \mathrm{~mm}$ from $0.206 \pm 0.005 \mathrm{~mm}$ ) (Fig. 3). By topical application, ginkgetin significantly inhibited ear edema (22.8, $30.5 \%$ inhibition at $20,80 \mu \mathrm{g} / \mathrm{ear} /$ treatment, respectively) (Fig. 3a). Prednisolone (10 $\mu \mathrm{g} /$ ear/treatment) showed potent inhibition of ear edema $(66.3 \%)$. Using the ear biopsies, PG levels were checked. As expected, TPA treatment drastically increased $\mathrm{PGE}_{2}$ concentration in the lesion $(24.8 \pm 2.1 \mathrm{ng}$ / biopsy) from the basal level (3.4 $\pm 0.3 \mathrm{ng} /$ biopsy). Ginkgetin moderately reduced $\mathrm{PGE}_{2}$ concentration $(30.2-31.1 \%)$, while prednisolone showed a slightly higher inhibition of $\mathrm{PGE}_{2}$ production $(36.5 \%)$.

For elucidating the effects on proinflammatory gene expression, RT-PCR was employed using the ear biopsies. TPA treatment considerably induced the expression of COX-2 and IL-1 $\beta$ genes (Fig. 4). Other inducible genes of ICAM-1 and TNF- $\alpha$ were very weakly induced whereas iNOS mRNA was not detected. The constitutive genes, COX-1 and fibronectin, were constantly expressed as expected, but there was some increase of mRNA expression by 7-d TPA treatment. Under this condition, ginkgetin dose-dependently inhibited IL- $1 \beta$ expression among the inducible genes tested, but not statistically significant $(16.6,50.9 \%$ inhibition at low and high dose treatment, respectively). COX-2 expression was weakly reduced only by high dose treatment of ginkgetin $(13.7 \%$ inhibition). The changes of ICAM-1 and TNF- $\alpha$ gene expression by ginkgetin were not meaningful since expression levels of these two genes were too low. On the other hand, prednisolone potently inhibited COX-2 and IL- $1 \beta$ expression (75.7, 95.7\% inhibition, respectively).

For toxicity evaluation, ginkgetin was applied to the dorsal 

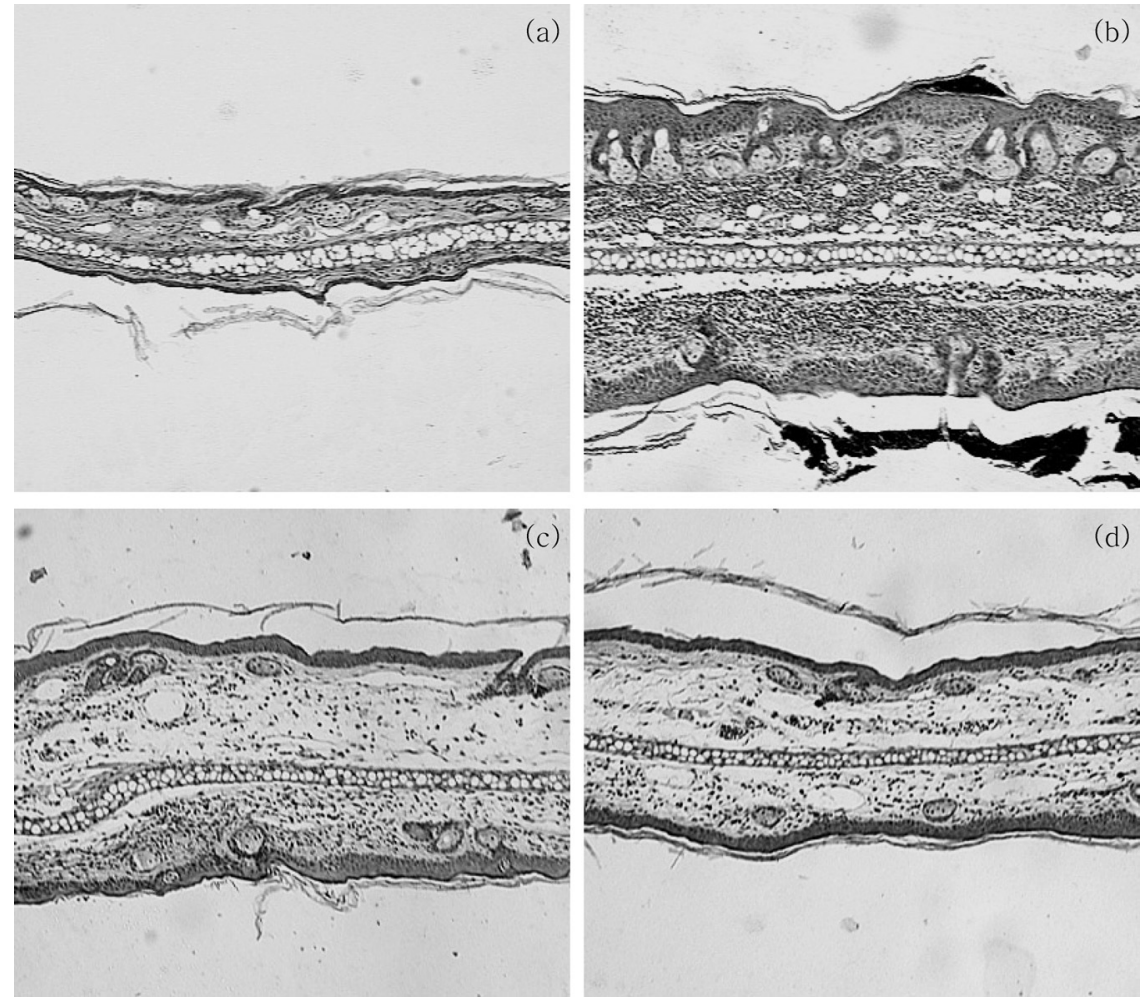

Fig. 2. Cross Sections of ICR Mouse Ear

(a) Non-treated, (b) TPA, (c) TPA+ ginkgetin ( $80 \mu \mathrm{g} /$ ear/treatment), (d) TPA + prednisolone $(10 \mu \mathrm{g} /$ ear/treatment). $\times 100$.
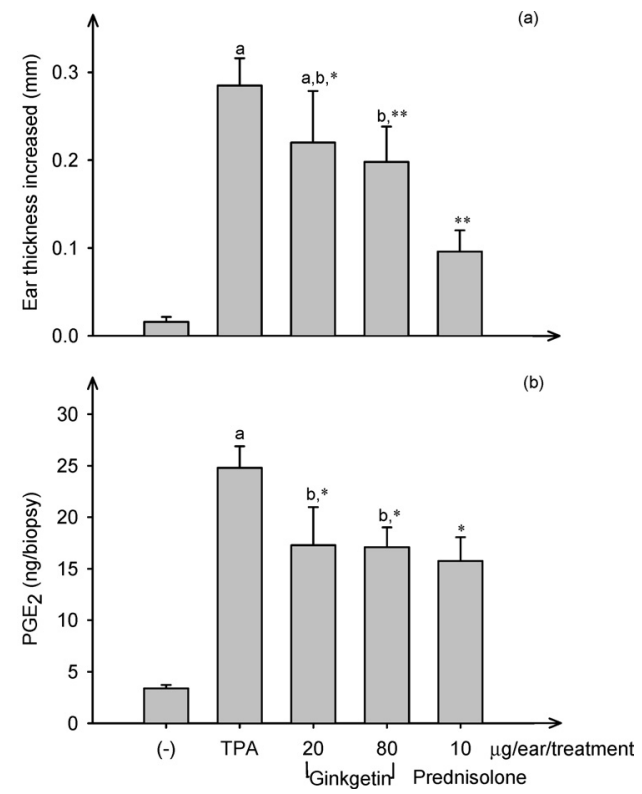

Fig. 3. Effects of Ginkgetin on Ear Edema and $\mathrm{PGE}_{2}$ Production

(a) Inhibition of TPA-induced ear edema $(n=5)$, (b) inhibition of TPA-induced PGE production $(n=4)$. a,b Different alphabets represent the groups of different each other by ANOVA followed by Duncan test $(p<0.05)$. $* p<0.1, * * p<0.05$, significantly different from the TPA-treated group by Student's $t$-test.

area of SKH-1 hairless mice ( $400 \mu \mathrm{g} /$ mouse $/ \mathrm{d}, 5 \mathrm{~d}$ a week). After 3 months, any apparent toxicity including appearance difference and differences of body weights and major organ weights was not found (data not shown). All these findings indicate that ginkgetin possesses inhibitory activity against chronic skin inflammation and may be safely used in long- term topical treatment.

\section{DISCUSSION}

This investigation has clearly proven that ginkgetin inhibited the inflammatory responses of an animal model of chronic skin inflammation. Depending on animal models of skin inflammation employed, there are substantial differences in the nature of inflammation produced. For instance, phenoltreated contact dermatitis in mice produced an acute inflammation accompanied by dermal edema, and an animal model of delayed hypersensitivity induced an infiltration of inflammatory cells in the lesion. ${ }^{19,20)}$ Multiple TPA treatment used in this study, on the other hand, provoked the characteristic changes such as edema and epidermal keratinocyte proliferation. These results were well matched with other's finding. ${ }^{21)}$ In particular, ginkgetin reduced the epidermal hyperplasia.

Previously, it was found that multiple treatment of TPA for 3 consecutive days (3-d model) potently induced several proinflammatory genes including COX-2, IL- $1 \beta$, TNF- $\alpha$ and ICAM-1 on ICR mouse ear. ${ }^{22)}$ Compared to $3-\mathrm{d}$ model, COX-2 mRNA expression was weaker, and the degree of COX-2 and IL- $1 \beta$ expression had larger variation within the animals of the same group. The expression levels of ICAM-1 and TNF- $\alpha$ expression were minimum in 7-d model, while IL-1 $\beta$ gene expression was prominent on both models. It is thought that a shift to chronic response in 7-d model from 3d model may provide these changes, suggesting that IL- $1 \beta$ expression is likely more important than those of $\mathrm{COX}-2$, ICAM- 1 and TNF- $\alpha$. Ginkgetin was found to inhibit IL- $1 \beta$ expression. However, it is not certain why there was some increase of COX-1 and fibronectin mRNA levels by TPA treat- 

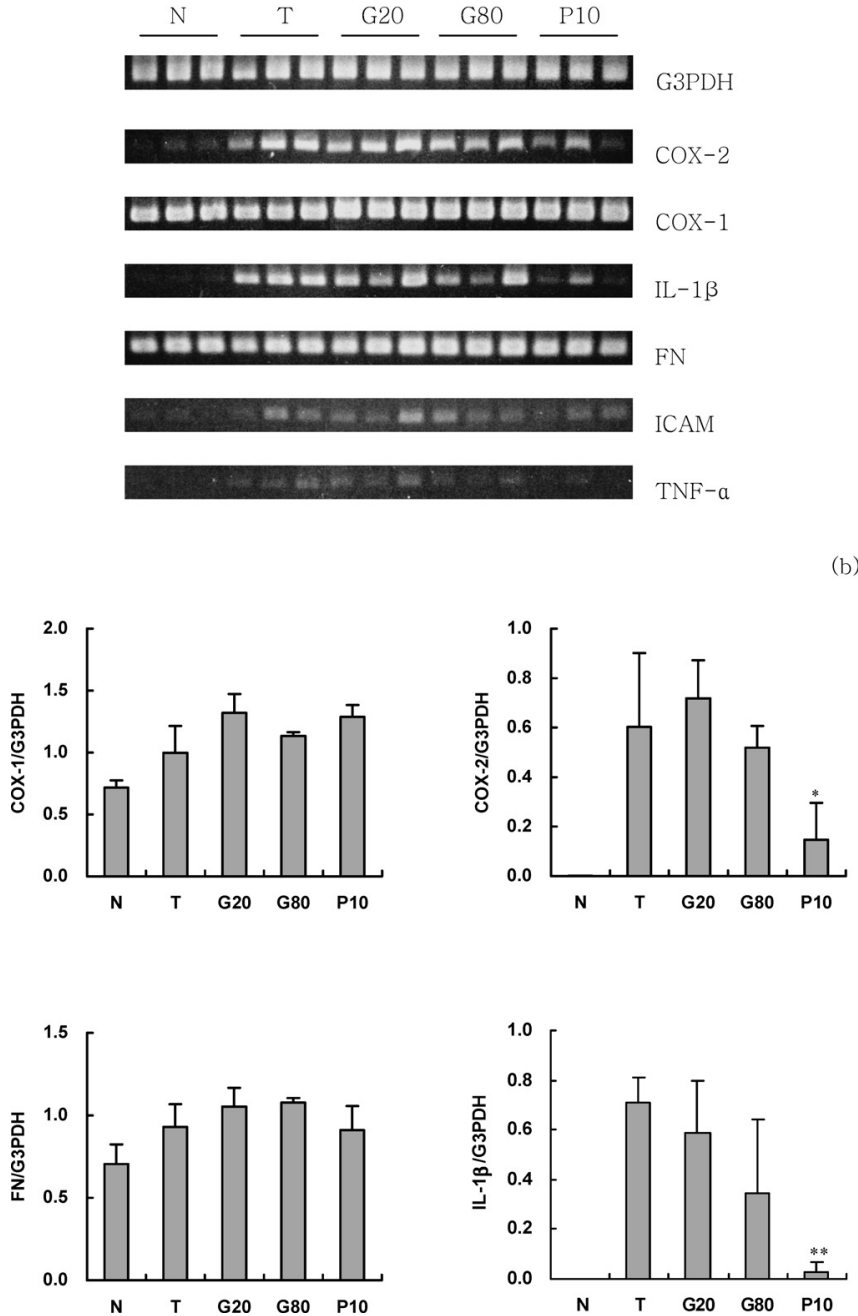

Fig. 4. Effects of Ginkgetin on Proinflammatory Gene Expression

(a) RT-PCR analysis. N: non-treated, T: TPA, G: TPA+ginkgetin $(20,80 \mu \mathrm{g} /$ ear/treatment), P: TPA+prednisolone $(10 \mu \mathrm{g} / \mathrm{ear} /$ treatment $)$. (b) Density ratios. $* p<0.1$, $* * p<0.05$, significantly different from the TPA-treated group by Student's $t$-test $(n=3)$.

ment with/without ginkgetin.

Especially, on 3-d model, ginkgetin clearly reduced COX2 induction and $\mathrm{PGE}_{2}$ production in the skin lesion. ${ }^{22)}$ On the contrary, in the present 7-d model, ginkgetin did not show the meaningful reduction of COX-2 expression. Furthermore, ginkgetin was previously found to show no effect on COX-1 and COX-2 activities. ${ }^{16}$ Therefore, an inhibition of COX-2 expression or COX activity may not be responsible for the reduction of $\mathrm{PGE}_{2}$ concentration by ginkgetin treatment. Instead, it is speculated that the some reduction of $\mathrm{PGE}_{2}$ concentration may be at least partly due to $\mathrm{PLA}_{2}$ inhibition and/or inhibition of IL- $\beta$ expression by ginkgetin. This speculation may be supported by the previous finding that the certain biflavonoids including ginkgetin were revealed as PLA $_{2}$ inhibitors. ${ }^{14,15,23)}$ It is also known that IL- $1 \beta$ enhances prostanoid production. ${ }^{24,25)}$ But the detailed mechanism needs to be further elucidated.

To present, there is a difficulty to successfully treat chronic skin inflammatory disorders. Therefore, it is desirable to develop anti-inflammatory agents having different cellular action mechanism(s) from those of conventional drugs such as topical steroids. In this respect, ginkgetin has a potential for new anti-inflammatory agent since this compound is a multifunctional agent, attenuating proinflammatory gene expression as well as inhibiting PLA $_{2}$. Ginkgetin may be beneficial against chronic skin inflammatory disorders such as AD by topical application.

Acknowledgements This work was supported by grant No. R01-2004-000-10134-0 from the Basic Program of the Korea Science \& Engineering Foundation.

\section{REFERENCES}

1) Andersen S., Sjursen W., Laegreid A., Volden G., Johansen B., Inflammation, 18, 1-12 (1994)

2) Fogh K., Kragballe K., Prostag. Other Lipid Mediators, 63, 43-54 (2000).

3) Kampfer H., Brautigan L., Geisslinger G., Pfeilschifter J., Frank J., J. Invest. Dermatol., 120, 880-890 (2003).

4) Lee J. L., Mukhtar H., Bickers D. R., Kopelovich L., Athar M., Toxicol. Appl. Pharmacol., 192, 294-306 (2003).

5) Veale D., Rogers S., Fitzgerald O., Br. J. Dermatol., 132, 32-38 (1995).

6) LaDucca J. R., Caspari A. A., Dermatol. Clin., 19, 617—635 (2001).

7) Bruch-Gerharz D., Fehsel K., Suschek C., Michel G., Ruzicka T., Kolb-Bachofen V., J. Exp. Med., 184, 2007-2012 (1996).

8) Bruch-Gerharz D., Ruzicka T., Kolb-Bachofen V., J. Invest. Dermatol., 110, 1-7 (1998).

9) Amella M., Bronner F., Hagg M., Anton R., Landry Y., Planta Med., 1985, 16-20 (1985).

10) Kim H. P., Mani I., Iversen L., Ziboh V. A., Prostag. Leukot. Essen. Fatty Acids, 58, 17-24 (1998).

11) Selvam C., Jachak M., J. Ethnopharmacol., 95, 209-212 (2004).

12) Banerjee T., Valacchi G., Zibo V. A., van der Vliet A., Mol. Cell Biochem., 238, 105-110 (2002).

13) Baek S. H., Yun S. S., Kwon T. K., Kim J. R., Chang H., Kwak J. Y., Kim J. H., Kwun K. B., Shock, 12, 473-478 (1999).

14) Chang H. W., Baek S. H., Chug K. W., Son K. H., Kim H. P., Kang S. S., Biochem. Biophys. Res. Commun., 205, 843 -849 (1994).

15) Kim H. P., Pham H. T., Ziboh V. A., Prostag. Leukot. Essen. Fatty Acids, 65, 281-286 (2001).

16) Kwak W.-J., Han C. K., Son K. H., Chang H. W., Kang S. S., Park B. K., Kim H. P., Planta Med., 68, 316-321 (2002).

17) Kang S. S., Kim J. S., Kwak W.-J., Kim K.-H., Kor. J. Pharmacogn., 21, 111-120 (1990).

18) Stanley P. L., Steiner S., Havens M., Tramposch K. M., Skin Pharmacol., 4, 262-271 (1991).

19) Mitsui G., Mitsui K., Hirano T., Ohara O., Kato M., Niwano Y., Immunol. Lett., 68, 191-197 (2003).

20) Lim H., Park H., Kim H. P., Arch. Pharm. Res., 27, $442-448$ (2004).

21) Giannaras A., Selig W., Ellis J., Hullinger T., Eur. J. Pharmacol., 506, 265-271 (2005).

22) Chi Y. S., Lim H., Park H., Kim H. P., Biochem. Pharmacol., 66, $1271-1278$ (2003).

23) Gil B., Sanz M. J., Terencio M. C., Gunasegaran R., Paya M., Alcaraz M. J., Biochem. Pharmacol., 53, 733-740 (1997).

24) Hseu Y.-C., Wu F.-Y., Wu J.-J., Chen J.-Y., Chang W.-H., Lu F.-J., Lai Y.-C., Yang H.-L., Int. Immunopharmacol., 5, 1914-1925 (2005).

25) Moolwaney A. S., Igwa O. J., Mol. Brain Res., 137, 202-212 (2005). 